\title{
Planning for Crew Exercise for Deep Space Mission Scenarios
}

\author{
E. Cherice Moore \\ Johnson Space Center \\ 2101 NASA Parkway \\ Houston, TX 77058 \\ (281) 483-8780 \\ cherice.moore-1@nasa.gov
}

\author{
Dr. Jeff Ryder \\ Universities Space Research \\ Association \\ 2101 NASA Parkway \\ Houston, TX 77058 \\ (281) 244-0968 \\ jeffrey.ryder-1@nasa.gov
}

\begin{abstract}
Exercise which is necessary for maintaining crew health on-orbit and preparing the crew for return to $1 \mathrm{G}$ can be challenging to incorporate into spaceflight vehicles. Deep space missions will require further understanding of the physiological response to microgravity, understanding appropriate mitigations, and designing the exercise systems to effectively provide mitigations, and integrating effectively into vehicle design with a focus to support planned mission scenarios. Recognizing and addressing the constraints and challenges can facilitate improved vehicle design and exercise system incorporation.
\end{abstract}

\section{TABLE OF CONTENTS}

1. INTRODUCTION ....................................................1

2. EXERCISE AND CREW HEALTH ..........................1 3. EXERCISE DRIVERS FOR DEEP SPACE

MISSIONS ..............................................................4

4. DESIGNING VeHICLES TO SUPPORT EXERCISE6

5. CHALLENGES FOR IMPROVING INTEGRATION OF EXERCISE INTO DEEP SPACE VEHICLES .........7

6. CONCLUSION.......................................................7

REFERENCES...............................................................7

BIOGRAPHY .........................................................

\section{INTRODUCTION}

One of the major challenges for deep space exploration will be dealing with changes to human physiology that are associated with reduced gravitational environments. The human body experiences multiple detrimental changes that require a suite of exercise hardware capability to mitigate. The primary physiological systems involved that are targets for mitigation via exercise countermeasures include the musculoskeletal system (both muscle and bone) and the cardiovascular system as it relates to aerobic capacity and work performance. Muscle atrophy, bone loss, and reduced aerobic capacity all occur in response to space-flight and exercise is a recognized operational countermeasure that continues to mature through research and spaceflight experience. A range of challenges exists from providing the needed physiological mitigations with a robust and optimized exercise system to designing the vehicle to adequately incorporate the exercise system. While there are common threads across all missions in terms of physiological protocol and hardware requirements, there are also specific needs and hurdles which vary across different deep space mission scenarios.

\section{EXERCISE AND CREW HEALTH}

To understand the hardware and vehicle designs necessary to implement the appropriate exercise systems, insight into the physical deconditioning that occurs is necessary. Spaceflight-induced physical deconditioning increases the risk of reduced crewmember performance and thus the likelihood of impacts to mission success and crew member safety. In some cases, spaceflight-induced deconditioning has the potential to affect the long-term health of the astronaut. These risks were recognized early on in the space program. Exercise countermeasures capabilities have been available since even the Gemini missions and requirements for crew to perform exercise have existed since the Space Shuttle Program.

Skeletal muscle is a highly adaptable tissue that responds to its environment and the stresses imposed upon it. Unloading of the postural muscles such as those in the lower limbs and back results in atrophy during spaceflight. While muscle exhibits an ability to readapt when functional overload is again applied to it, the effects are not immediate. Therefore, loss of muscle mass, strength and power will all present the greatest risk to crew performance and safety upon early introduction back into any planetary environment with significant gravitational forces. This would be especially true of a Mars landing or a return to Earth where not only are the new gravitational challenges the greatest, but the potential demands may also be at their apex. For instance, landing on Mars may require some degree of early surface extravehicular activity (EVA) in order to establish a functioning surface habitat. Of greatest concern would be whether or not crew could maintain their functional strength sufficient to deal with an emergency situations such as the need for an unassisted contingency egress; this would be the case for both a landing on Mars as well as during the return to Earth following deep space exploration. Partial gravity environments such as Mars should not be expected to provide sufficient protection of muscle mass; at least not to 
a degree any greater than that environment represents (e.g. a 3/8 g should not be expected to maintain muscle mass to Earth levels). Without countermeasures, this could result in unexpected challenges during normal surface EVA operations on Mars even with sufficient time to adapt to the partial g environment.

Space flight and research analogs for space flight such as head-down-tilt have given us the greatest insights in to what to expect in terms of deconditioning during deep space missions. Each of these approaches has its strengths and weaknesses. Spaceflight itself obviously is the truest indicator of spaceflight-induced muscle atrophy. One factor confounding the results though is the variability of the conditions. While nearly all missions since the start of the Shuttle have been supported by exercise countermeasures hardware, the types of hardware available, hardware reliability, exercise protocols, mission duration and even crew member nutrition vary from mission to mission and Program to Program. While the bed rest platform cannot fully simulate the unloading aspect to in an identical manner to space flight there are a number of key aspects that aid our understanding in a way that even retrospective space flight data does not. Bed rest studies allow for very tight control of the conditions that the subjects experience including duration, nutrition, sleep, the countermeasures hardware being examined and even the details of experimental protocols aimed at improving efficacy in reducing unloading-induced atrophy. The bed rest platform also provides an opportunity to determine the true deconditioning baseline where no countermeasures are implemented as this approach will not occur in crew members in flight.

A clearly defined timeline for muscle atrophy and loss of strength during space exploration has yet to be established. Nevertheless, existing data show that significant losses can occur during even short duration space flight. Evidence for this is provided from Space Shuttle missions which typically were in the 8-17 day range. Loss of muscle strength and mass were readily observable by various techniques including isokinetic dynamometry for strength [1], and magnetic resonance imaging (MRI) [2], single muscle fiber diameter measures [3, 4], and histochemistry [5] on muscle cross sections from muscle biopsy preparations. What is clear is that data from Shuttle versus Space Station observations indicate that muscle atrophy progresses well past the short duration spaceflight timeline. MRI data from crew aboard Space Shuttle for 17 days showed a reduction of $7.4 \%$ in quadriceps muscles on landing day with reductions of the gastrocnemius and soleus muscle of the calf by $12.4 \%$ and $9.8 \%$, respectively [2]. Following stays of 4 to 7 months on Space Station Mir, decrements in quadriceps (12.1\%), gastrocnemius (23.8\%) and soleus (19.6\%) muscles were considerably larger [2]. Similar measures have also been performed following 6-month missions aboard the International Space Station (ISS) [6, 7]. Decrements in ISS crew were shown to be less than those presented above for Space Station Mir with reductions reported as 5.9\%, 10-10.3\%, and 15-18.6\% for quadriceps, gastrocnemius, and soleus, respectively. The ISS muscle volume data were measured 4-6 days post-landing and there is evidence to suggest that part of the apparent improvement between Mir and ISS could be due to the timeline of analysis, nevertheless, it is important to note that ISS crew had access to a resistance training hardware. This also undoubtedly contributes to the improved mitigation of muscle loss.

Bed rest studies also prove out the progressive manner of muscle loss in unloading. Muscle loss has been reported to progress from 10 to $19 \%$ between days 29 and 89 in quadriceps muscle and $16 \%$ to $29 \%$ in soleus muscle over that same period of time [8]. Those bed rest results, while shorter in duration, involved no countermeasures, whereas ISS data are from crew that had access to daily countermeasures involving aerobic and resistance exercise, albeit it on arguably with sub-optimal resistance exercise loads. The resistance exercise hardware that those particular crewmembers had access to was the interim resistive exercise device (iRED). The iRED was an elastomer-based resistance exercise machine with a maximum loading capacity of 300 pounds. In order to perform whole-body exercises such as a squat, resistance exercise machines in space must also be capable of replacing body weight that is absent in microgravity. Because of this, 300 pounds is functionally less than one would intuitively think based on Earth experience. The iRED actually suffered from inadequate load capacity making it possible for crew to max out the system. With the delivery of the Advanced Resistive Exercise Device (ARED) to ISS, crew now have a resistance load of up to 600 pounds. While efficacy data for muscle mass and strength since the deployment of ARED are not available at this time, preliminary data for lean body tissue as assessed by dual x-ray absorptiometry (DXA) indicate improved outcomes (total lean body mass, presumably primarily muscle) simply by the availability of increased loads allowing for more maximal contractions to be performed during resistance exercise [9]. High resistance loads imparted by via inertial flywheels have been effective in preventing muscle atrophy. Performance of resistance exercise involving maximal contractions during 89 days of bed rest completely protected muscle mass in quadriceps muscles and the atrophy significantly attenuated in calf muscles [8]. Thus, hardware countermeasures that are capable of delivering high loads to the subject are likely to have the greatest effectiveness in maintaining muscle performance for long duration space flights.

Maximal aerobic capacity $\left(\mathrm{VO}_{2 \max }\right)$ is the maximal rate at which one can utilize oxygen to convert physiological fuel sources into energy. $\mathrm{VO}_{2 \max }$ is therefore one of the fitness gold standards used in both research and sports performance laboratories. Similar to skeletal muscle size and strength, aerobic capacity $\left(\mathrm{VO}_{2 \max }\right)$ readily responds to varied states of training and detraining. Changes in $\mathrm{VO}_{2 \max }$ will positively and negatively impact endurance type performance making the protection of $\mathrm{VO}_{2 \max }$ a priority for 
deep space missions. As was observed for muscle morphology and performance, $\mathrm{VO}_{2 \max }$ has also been shown to be negatively impacted by during both short and long duration space flight. $\mathrm{VO}_{2 \max }$ was assessed during and immediately following flight on 6 crew members who flew either 9 or 14 day shuttle mission [10]. Respiratory gasses were collected on crewmember subjects as the performed a graded exercise test on a bicycle ergometer where workloads were gradually increased until termination due to volitional fatigue. Values for $\mathrm{VO}_{2 \max }$ remained at pre-flight levels during the in-flight measurements (5 to 8 days into the flight), however immediate measures following landing revealed a $22 \%$ reduction in $\mathrm{VO}_{2 \max }$ and a $17 \%$ in the corresponding power output. The interpretation of these results was that, at least during short duration missions, that that cardiovascular system can compensate for a loss of spaceflight-induced blood volume in a gravity-free environment to maintain sufficient cardiac output to keep $\mathrm{VO}_{2 \max }$ at pre-flight levels.

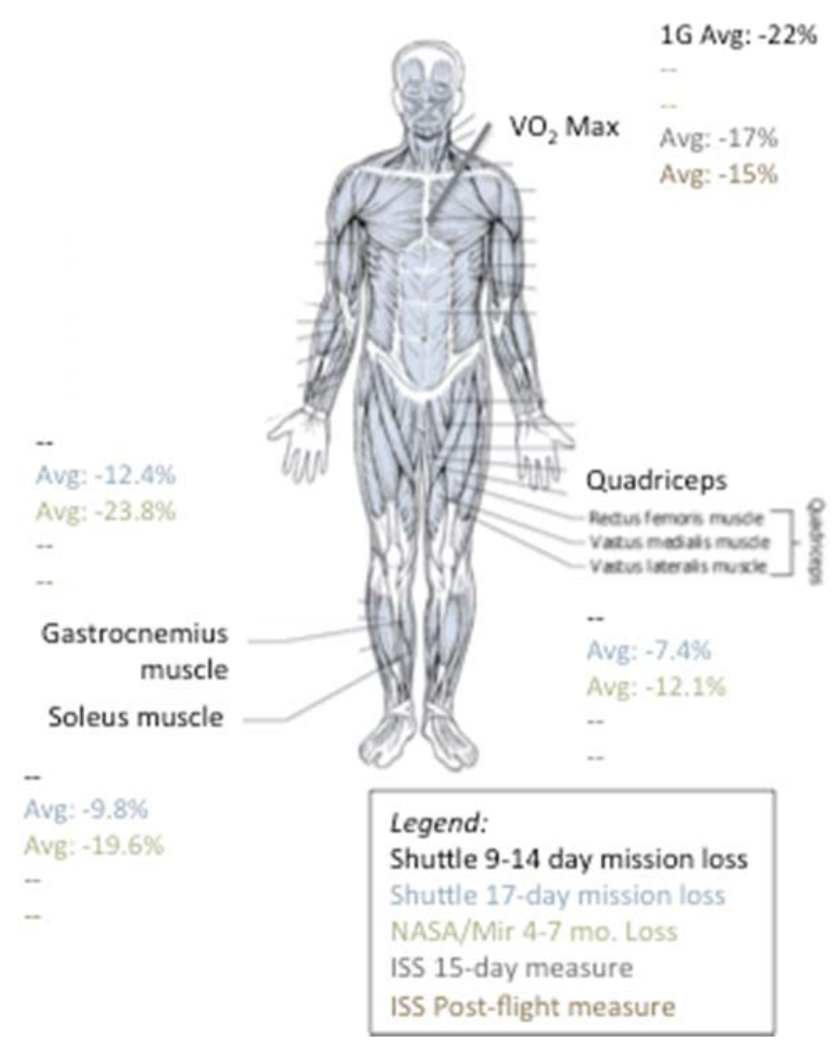

Figure 1 - Illustration showing muscle and aerobic losses

A recent publications on the effects of long duration ISS spaceflight on inflight and post-flight $\mathrm{VO}_{2 \max }$ may be even more informative on what to expect the effects of deep space mission to by on aerobic capacity [11]. Fourteen ISS crewmembers had their aerobic capacity measures in a manner similar to that described above for Space Shuttle mission. Measurements were preformed pre-flight, in-flight (flight day 15 and every 30 days thereafter), and within 1-2 days of return. In contrast to findings at days 5-8 during shuttle missions, mean values from crew after 15 days of spaceflight aboard ISS reveal inflight decrements of $\mathrm{VO}_{2 \max }$ (17\%) and the corresponding power out (24\%). Subsequent inflight measures did show gradual improvements in $\mathrm{VO}_{2 \max }$ over the 6 month mission though levels did not fully reach pre-flight values. As with Space Shuttle mission, early postflight landing measurements for ISS crew revealed a returnto-earth decrement with values dropping to levels $15 \%$ below preflight status. The authors point out that while mean values were reduced for the cohort that was analyzed, 4 crewmembers who performed aerobic exercise at higher intensities actually were able to maintain post-flight $\mathrm{VO}_{2 \max }$ values to pre-flight standards. This reiterates the lessons learned from spaceflight and the protection of muscle mass, that providing hardware that allows for intense exercise will be key to maximizing he effectiveness of exercise countermeasures.

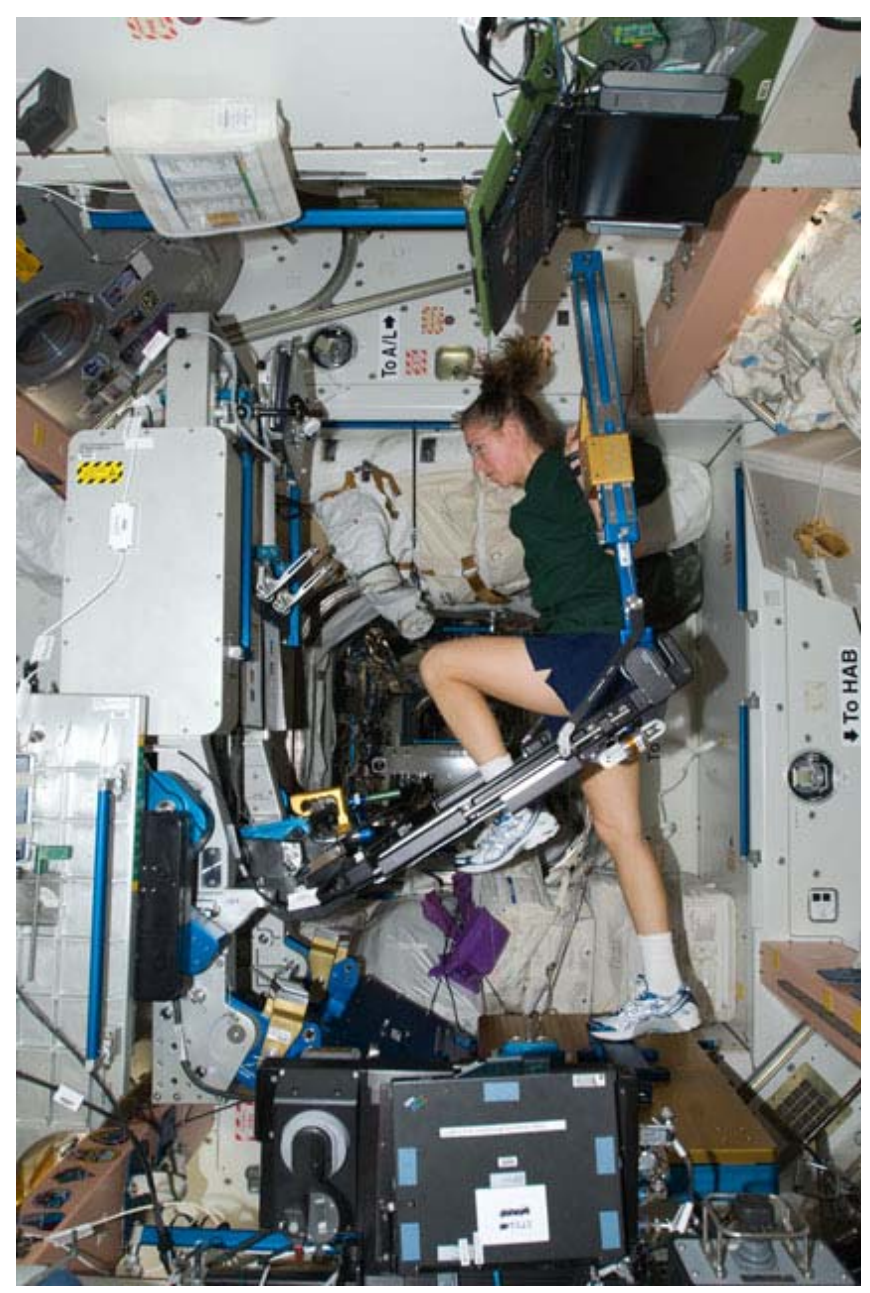

Figure 2 - ISS Advanced Resistive Exercise Device (ARED) being used for single leg squats. iss018e025461

Maintenance of bone health will also be a challenge for deep space exploration. Gravitational unloading promotes bone resorption which under chronic conditions may elevate risks for fracture or early onset osteoporosis. Unlike muscle and aerobic capacity concerns, effects are considerably 
more limited during short duration flights. Post-flight bone mineral density during Space Shuttle missions is unaltered at the resolution for which DXA could provide at the time [12]. While these types of short missions (less than 3 weeks) may not be of great concern as it relates to risks associated with bone remodeling, the same is certainly not the case in response to long duration space flight. Like muscle, however, the key to maximizing the effectiveness of exercise countermeasures appears to involve providing sufficient load. Bone loss was compare in ISS crew who had access to iRED (low load capability) versus those who had access to ARED (high load capability) [13]. In crew who had access to iRED bone mineral as assessed by DXA was reduced approximately $5 \%$ the femoral neck, trochanter and total hip. Deficits in bone loss in these areas were attenuated by about $50 \%$ in crew with access to ARED. Similar trends were observed for the pelvis and lumbar spine.

\section{EXERCISE DRIVERS FOR DEEP SPACE MISSIONS}

Multiple aspects about exercise will influence what capability is needed for any given space flight mission. Integrating the physiological performance needs with the mission concepts such as the number of vehicles involved, transit times, planetary activities, landing scenarios, operational approaches, mission environments and tasks, will be necessary to scope the needed exercise system suite of hardware. As indicated above, intensity will be a key component for exercise countermeasures protocols moving forward for deep space exploration. This can be expected for both resistance and aerobic exercise countermeasures protocols. Higher intensity exercise protocols often translate into faster movements and/or higher loads for resistive exercises, higher speeds for treadmill-based exercises, and increased resistance in rowing and cycling-based exercises. Therefore, in addition to hardware that can provide sufficient workloads, the integration of the exercise system into the vehicle will also need to sustain the impacts of performing such workloads. Some of the basic performance parameters for resistive exercise includes providing exercise loads from 20 to 600 lbs. with highly accurate delivery, supporting any combination of movement patterns of triple extension, vertical pushing and pulling, and horizontal pushing and pulling, loading profiles including both constant and inertial loading, provide at least 1:1 eccentric to concentric loading, and be easy to use. Aerobic exercise performance parameters include providing an average power loading of $400 \mathrm{~W}$ with the ability to peak at $750 \mathrm{~W}$ when measured in 30-minute intervals. For treadmill activities maximum belt speeds of $12 \mathrm{mph}$ are desired. For cycling activities the hardware should support 120 revolutions per minute. For rowing activities, up to 75 strokes per minute is desired.

Both crew and hardware monitoring is often included with exercise systems to better protect for the health of both.
Crew monitoring may include loads, frequencies, cycle counts, durations, and heart rate monitoring. Hardware monitoring utilizes the same data for crew monitoring along with power performance, thermal measurements, and specific subcomponent monitoring such as dynamic mechanical items. Information needed for research studies may add additional monitoring capabilities (such as accelerometers, positional detectors, or footfall force loading profiles) or require increasing the data capture frequencies.

The overall mission concept which includes for example what vehicles will be used, overall mission duration, possible microgravity Extravehicular Activities (EVAs) or planetary EVAs, operational scheduling approach, and vehicle reentry and landing scenarios all inform the number and type of exercise hardware needed. The mission duration actually has minimal impact on exercise drivers for deep space missions from a physiological perspective. Even for short missions (e.g. 8-21 days) NASA researchers know from experiences that there will be significant losses in aerobic and muscular fitness. Longer missions create greater deficits and increase risks to mission success and crew safety if left unmitigated. However, duration of mission has little impact on planning needs. Successful, countermeasures protocols for shorter missions will require high resistance / high load capabilities just as they will for longer missions. While protection of bone health may not be as great of concern for short missions as for long, a primary countermeasures approach involves resistance exercise. Because this is shared approach with the prevention of muscle atrophy, this is not a place for mass/volume savings for short versus long duration missions. One possible exception during short missions may be the need for a treadmill. Treadmills provide both an aerobic benefit for the cardiovascular system and a loading benefit for the skeletal system. Considering that short durations flights are expected to have minimal impacts to bone health, other modes of aerobic exercises can more could optionally be implemented instead of a treadmill.

Mission duration, however, does drive the level of robustness of the exercise system. The engineering approach required to create an exercise system with a high confidence level of reliability for a 20 day mission will be different than the approach to develop a system within the same confidence level of reliability for a 2 year mission. In both cases the system would need to be assessed for the ability to meet the needed cycle life, structural fatigue, and appropriate component wear evaluations. As the longer duration missions will see correspondingly greater cycles and more wear, materials and parts included should also be selected to withstand the additional life with minimal maintenance. Special attention to the design of the dynamic mechanical parts of the hardware can also increase the system's robustness.

Mission tasks and landing concept(s) inform the absolute performance needed from any given crewmember to achieve 
mission success. Mission tasks that drive exercise system performance needs can include endurance and strength to perform EVA activities, strength required for hatch openings, or the sensorimotor adaptation, strength and endurance needed to exit the vehicle in nominal and offnominal situations.

The operational scheduling approach informs how much of the day is available to schedule crew usage of the exercise system. Using that time and incorporating any other scheduling constraints (such as no exercise during planned sleep schedule or immediately after meals) and then dividing the schedule into exercise slots so as to accommodate the needed exercise time for each crew gives insight into the maximum number of crew that can use any given exercise hardware during the mission. For example, using ISS scheduling constraints (See Table 1), and assuming that a single exercise hardware system can be developed that provides all aerobic, resistive, bone, and sensorimotor countermeasures needed, the maximum number of crew that one device could support per mission is four. (See Table 2) Once missions are planned to be greater than 4 crew and additional exercise hardware is needed, decisions may be evaluated on whether capabilities should be separated (such as using separate resistive exercise hardware and a treadmill) or a second all-capable device would be flown. Each new exercise hardware would require the additional crew and hardware volume to allow concurrent exercise activities and not impede other nearby crew operations.

Table 1: Exercise Timelining Assumptions

\begin{tabular}{|l|l|}
\hline Activity & $\begin{array}{l}\text { Assumed } \\
\text { Duration } \\
\text { (hrs) }\end{array}$ \\
\hline Sleep & 8.5 \\
\hline Post-sleep \& Meal & 1.5 \\
\hline $\begin{array}{l}\text { Lunch (assumes } 30 \text { minutes overlap for all } \\
\text { crew) }\end{array}$ & 1 \\
\hline Pre-sleep \& meal & 2 \\
\hline Workday & 11 \\
\hline Time after meals before exercising permissible & 0.5 \\
\hline $\begin{array}{l}\text { Assumes one exercise day off for each crew per } \\
\text { week }\end{array}$ & 1 \\
\hline $\begin{array}{l}\text { Assumes } 1 \text { hour aerobic exercise per crew for 6 } \\
\text { of } 7 \text { days (includes 30 minutes for clothing } \\
\text { changes, hardware setup, shutdown and cleanup } \\
\text { time) }\end{array}$ & 1 \\
\hline $\begin{array}{l}\text { Assumes } 1.5 \text { hours resistive exercise per crew } \\
\text { for } 6 \text { of } 7 \text { days (includes 30 minutes clothing }\end{array}$ & 1.5 \\
\hline
\end{tabular}

changes, hardware setup, shutdown and cleanup time)

Table 2: Example One-Week Exercise Timeline

Crew exercise timeline example for four crew where "A" \& "R" indicate "aerobic" or "resistive"-focused workout \& number identifies which crew is exercising.

\begin{tabular}{|c|c|c|c|c|c|c|c|}
\hline Hour & $\begin{array}{c}\text { Day } \\
1\end{array}$ & $\begin{array}{c}\text { Day } \\
2\end{array}$ & $\begin{array}{c}\text { Day } \\
3\end{array}$ & $\begin{array}{c}\text { Day } \\
4\end{array}$ & $\begin{array}{c}\text { Day } \\
5\end{array}$ & $\begin{array}{c}\text { Day } \\
6\end{array}$ & $\begin{array}{c}\text { Day } \\
7\end{array}$ \\
\hline $8.5 \mathrm{hr}$ & \multicolumn{7}{|c|}{ Sleep } \\
\hline $1.5 \mathrm{hr}$ & \multicolumn{7}{|c|}{ Post-Sleep \& meal } \\
\hline $.5 \mathrm{hr}$ & \multicolumn{7}{|c|}{ Post-meal recovery } \\
\hline $1 \mathrm{hr}$ & A1 & & A1 & A1 & A1 & A1 & A1 \\
\hline $\begin{array}{l}1.5 \\
\text { hrs }\end{array}$ & $\mathrm{R} 2$ & R2 & & $\overline{\mathrm{R} 2}$ & $\mathrm{R} 2$ & $\mathrm{R} 2$ & R2 \\
\hline $1 \mathrm{hr}$ & A3 & A3 & A3 & & A3 & A3 & A3 \\
\hline $\begin{array}{l}1.5 \\
\text { hrs }\end{array}$ & $\mathrm{R} 4$ & $\mathrm{R} 4$ & $\mathrm{R} 4$ & $\mathrm{R} 4$ & & $\mathrm{R} 4$ & $\mathrm{R} 4$ \\
\hline $1 \mathrm{hr}$ & \multicolumn{7}{|c|}{ Lunch } \\
\hline $.5 \mathrm{hr}$ & \multicolumn{7}{|c|}{ Post-Lunch Recovery } \\
\hline $1.5 \mathrm{hr}$ & $\mathrm{R} 1$ & & $\mathrm{R} 1$ & $\overline{\mathrm{R} 1}$ & R1 & R1 & R1 \\
\hline $1 \mathrm{hr}$ & A2 & A2 & & A2 & A2 & A2 & A2 \\
\hline $1.5 \mathrm{hr}$ & R3 & R3 & R3 & & R3 & R3 & R3 \\
\hline $1 \mathrm{hr}$ & A4 & A4 & A4 & A4 & & A4 & A4 \\
\hline $2 \mathrm{hr}$ & \multicolumn{7}{|c|}{ Pre-sleep } \\
\hline $\begin{array}{l}\text { Crew } \\
\text { Day } \\
\text { off }\end{array}$ & & 1 & 2 & 3 & 4 & & \\
\hline
\end{tabular}

If the mission concept includes crew residence in multiple vehicles, each of those vehicles would need to be evaluated for the type of exercise required for the portion of the mission those vehicles represent. In some cases the vehicle habitable volume may be dual purposed to be used as an airlock for EVAs. If the exercise system is not otherwise protected, then the system would need to be designed to withstand the extreme temperature and pressure variations and still operate as intended upon return to habitable temperatures and pressures. 


\section{DESIGNING VeHICLES TO SUPPORT EXERCISE}

Once the mission concept and corresponding physiological exercise performance parameters have been identified, the vehicle integration planning and design can effectively move forward.

The location and amount of vehicle internal volume that will be needed for the exercise system tends to be the beginning approach for integrating exercise into a vehicle. A resistance exercise operating volume will need to provide enough room to safely and effectively accommodate a number of resistance exercise movements. Currently, squat, deadlift, and heel raise exercises compose the core movements for exercise countermeasures on the ISS, and these movements can be expected to be included in protocols in exploration missions. Each of these exercises involves a fully upright posture at some point in the movement (with the heel raise involving an upright on balls of feel posture that is slightly higher). Aerobic-focused exercise may include treadmill exercise, rowing, or cycle ergometry, and thus more than one scenario involving full body extension can be expected. The crew's operational volume uses the maximum defined anthropometry to assess the needed space. Additional volume may be added to include space for bouncing (such as during treadmill running), vibration isolation and stabilization (VIS) system dynamics, and any hardware protrusions around the crew operational space. The installed hardware volume includes not only the exercise device, but also the structural platforms, crew interfaces, VIS systems, avionics, cooling and needed power systems. Additional stowage volume may be required for items when not in use during exercise such as alternate exercise bars, crew treadmill harnesses, and any maintenance items that may be needed. The addition of the crew's operational volume, the installed hardware volume, and stowage volume would provide the total space needed for each exercise device. If multiple exercise devices are needed, that volume assessment would be done for each approach.

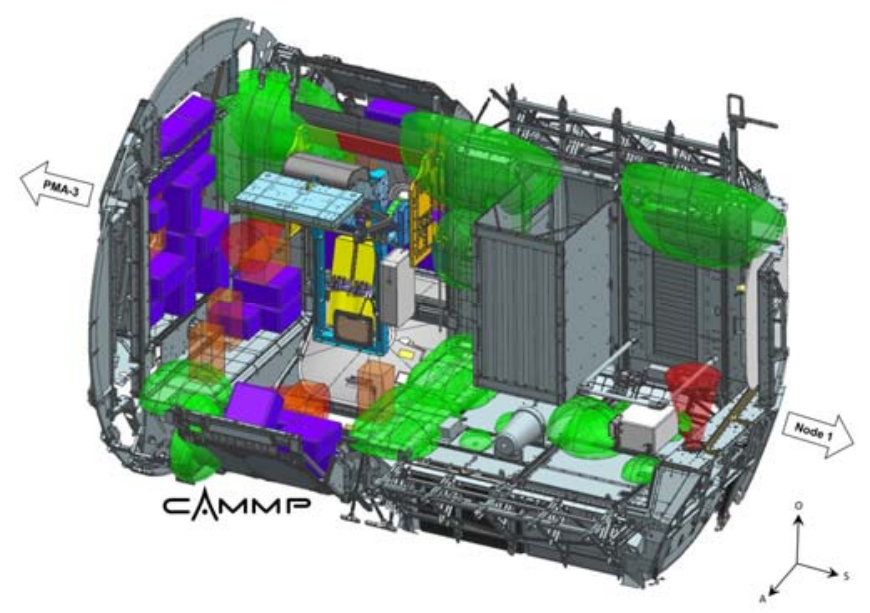

Figure 3 - ISS Node 3 exercise volume with stowage, ECLSS, and fire port accesses identified.

Placement of the exercise volume(s) within the vehicle should take into consideration the planned activities that are intended in the contiguous areas. Will exercise block translation paths? Would activities on nearby systems preclude exercise from occurring? Would exercise be in the line of sight for other video activities? Would the noise from the operations of the exercise hardware be disruptive to other activities? Will the location allow for efficient interfacing with the necessary vehicle systems? In limited vehicle habitable volume environments addressing all the locational concerns together becomes more challenging.

One of the vehicle interfaces that can be significant is the load impulses transmitted through the exercise system into the vehicle. Loads imparted back into the vehicle during exercise can also be a driver for vehicle design for deep space missions. Lifting, running rowing, cycling all involve a number of different forces that will be imparted into the exercise device and could be imparted into the vehicle itself at different magnitudes and vectors. These loads may need to be dampened or isolated in order to maintain the integrity of the vehicle. Past experience has shown that some exercise movements and frequencies can align with the vehicle's harmonic frequencies potentially creating a risk of severe damage to the vehicle if not isolated.

Vehicle power is often needed for exercise systems whether it is simply to capture exercise protocol data to transmit to the medical and engineering ground support teams or whether it is needed to run motors, cooling, avionics, and other systems such as those used in treadmills or operate control systems. Adequate thermal management should address both the metabolic heat loads and the exercise equipment thermal loading.

Metabolic loads will also impact vehicle design, In particular, metabolic loads occurring during aerobic exercise. Oxygen consumption, $\mathrm{CO}_{2}$ production, metabolic heat, and metabolic moisture will all need to be handled by an adequately sized environmental control system to support high intensity aerobic exercise. Ventilation needs to be positioned so as to facilitate needed airflow around the exercising crew member and around the exercise hardware as needed to cool the system.

Most spaceflight exercise systems have included some degree of data collection and bidirectional transmissions. The medical astronaut strengthening and conditioning teams work closely with the individual astronauts to provide customized exercise prescriptions that will provide the best mitigations. The system of prescription transmission in the form of a protocol, implementation, and then reporting back of actual activities constitutes some of the core drivers for integrating data and communications capabilities into the exercise systems. More advanced devices seamlessly integrate protocols and record the needed data for the medical, research and engineering teams which are then 
transmitted to the ground. For deep space missions where bandwidth is more limited, data will need to be optimized for transfer while still conveying the necessary information.

\section{CHALLENGES FOR IMPROVING INTEGRATION OF EXERCISE INTO DEEP SPACE VEHICLES}

As discussed in the previous sections, a number of factors must be considered in order to plan, design and integrate exercise systems into exploration vehicles. In vehicle design, habitable volume, launch mass, and systems integration tend to be major contributors to program costs, so efforts to improve performance in these areas are frequently sought after for all vehicle systems. Reliability is frequently desired as it drives the number of spares and maintenance items needed - all of which require stowage and vehicle upmass.

In order to improve overall vehicle mass and volume utilization, exercise systems also need to be designed to improve the mass:performance and volume:performance ratios. The ISS exercise systems require over 2,000 kg of equipment and impact more than $24 \mathrm{~m}^{3}$ of habitable volume. Efforts to use lighter-weight materials, improve design implementation, optimize volume usage while maintaining or improving the crew usage of the equipment are likely to reduce exploration vehicle costs.

Additional challenges to improve exercise integration include planning effective placement of the exercise system(s) within the vehicles. Both structural and operational considerations will need to be weighed and evaluated to determine the best possible locations.

Within all the challenges, a few aspects are unlikely to see much improvement. Crew exercise operational volume is primarily driven by the exercise movements and anthropometrics of the crews selected for flight. Even if novel movements and loading profiles are identified, the operational volume will still need to protect for the ranges of crew anthropometrics. Also, although research is being done to improve the time required for each crew to exercise, there may be a minimum exercise duration that will be needed if only to support the behavioral health of the crew.

\section{ConClusion}

The need for exercise to protect crew health is much more critical in the spaceflight environment. The vehicle designs for exploration deep space missions will need to include appropriate exercise systems effectively integrated into the vehicle structure. Future deep space missions such as those to send crews to an asteroid could be used as testing grounds for equipment expected to be used for longer duration exploration missions.

Exploration vehicle design for manned missions should adequately protect for the exercise system's mass, volume, power and other vehicle integration systems. When mission concepts change whether it is the overall mission duration, mission tasks, landing scenarios or integration with other vehicles, the applicability of the planned exercise system should be reevaluated and adjusted as necessary. Further work to improve the various subsystems of the overall exercise system is being pursued. Improvements in these areas will not only benefit future deep space missions, but can also benefit the public commercial exercise economy.

\section{REFERENCES}

[1] U.S. Munitions List, sections 38 and 47(7) of the Arms Export Control Act (22 U.S.C. 2778 and 2794(7).

[2] Aerospace Conference Web site: www.aeroconf.org 


\section{BIOGRAPHY}

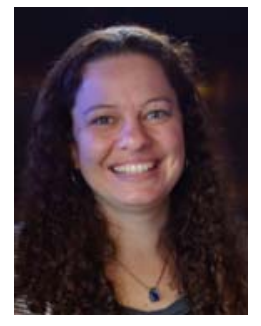

E. Cherice Moore, Advanced Countermeasures System Technology Development Manager, works within the NASA JSC Engineering Directorate to develop more efficient and compact exercise systems and improve integration into future spaceflight vehicles. Over Cherice's 18 year career, she has supported several projects and programs including the NASA/Mir Program, International Space Station's Human Research Facility project and the Crew Health and Countermeasures Systems. Her experience with exercise flight hardware includes being the NASA Project Manager for the Shuttle Cycle Ergometer, ISS's Cycle Ergometer with Vibration Isolation and Stabilization, Interim Resistive Exercise Device, BP-ECG, HRM, and $2^{\text {nd }}$ Treadmill exercise systems prior to transitioning into exploration-focused activities.

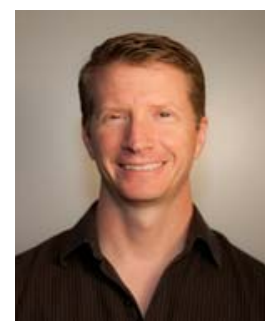

Jeffrey Ryder received a Ph.D. in Clinical Physiology from the Karolinksa Institute in Stockholm Sweden in 2000. Doctor Ryder has been employed since 2007 as a Senior Scientist with Universities Space Research Association in Houston, Texas. His position involves conducting research in the NASA Johnson Space Center Exercise Physiology and Countermeasures Laboratory. Research is focus and development of effective exercise countermeasures to protect muscle, bone and aerobic capacity during long duration space exploration missions. 
1. Day, M.K., et al., Adaptations of human skeletal muscle fibers to spaceflight. J Gravit Physiol, 1995. 2(1): p. P47-50.

2. LeBlanc, A., et al., Muscle volume, MRI relaxation times (T2), and body composition after spaceflight. J Appl Physiol, 2000. 89(6): p. 2158-64.

3. Widrick, J.J., et al., Effect of a 17 day spaceflight on contractile properties of human soleus muscle fibres. J Physiol, 1999. 516 ( Pt 3): p. 915-30.

4. Widrick, J.J., et al., Functional properties of slow and fast gastrocnemius muscle fibers after a 17day spaceflight. J Appl Physiol, 2001. 90(6): p. 2203-11.

5. $\quad$ Edgerton, V.R., et al., Human fiber size and enzymatic properties after 5 and 11 days of spaceflight. J Appl Physiol, 1995. 78(5): p. 1733-9.

6. Gopalakrishnan, R., et al., Muscle volume, strength, endurance, and exercise loads during 6-month missions in space. Aviat Space Environ Med, 2010. 81(2): p. 91-102.

7. Trappe, S., et al., Exercise in space: human skeletal muscle after 6 months aboard the International Space Station. J Appl Physiol (1985), 2009. 106(4): p. 1159-68.

8. $\quad$ Alkner, B.A. and P.A. Tesch, Knee extensor and plantar flexor muscle size and function following 90 days of bed rest with or without resistance exercise. Eur J Appl Physiol, 2004. 93(3): p. 294-305.

9. Smith, S.M., et al., Benefits for bone from resistance exercise and nutrition in long-duration spaceflight: Evidence from biochemistry and densitometry. J Bone Miner Res, 2012. 27(9): p. 1896906.

10. Levine, B.D., et al., Maximal exercise performance after adaptation to microgravity. J Appl Physiol (1985), 1996. 81(2): p. 686-94.

11. Moore, A.D., Jr., et al., Peak exercise oxygen uptake during and following long-duration spaceflight. J Appl Physiol (1985), 2014. 117(3): p. 231-8.

12. Ryder, J.W., R.A. Scheuring, and L.C. Shakelford, Musculoskelal Function, in Bionedical Results of the Space Shuttle rogram, D. Risin and P.C. Stepaniak, Editors. 2013, U.S. Government Printing Office: Washington D.C. p. 215-234.

13. Leblanc, A., et al., Bisphosphonates as a supplement to exercise to protect bone during long-duration spaceflight. Osteoporos Int, 2013. 24(7): p. 2105-14. 